\title{
Fit and Motivated: Outcome Predictors in Patients Starting a Program for Lifestyle Change
}

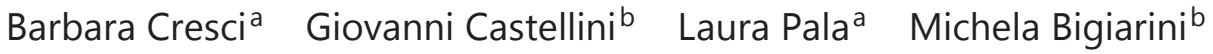 \\ Ester Romoli $^{b}$ Roberta Poggialic Cosetta Guarnieric \\ Barbara Biffi $^{b}$ Teresa La Ferlita ${ }^{c}$ Valdo Riccad ${ }^{d}$ Edoardo Mannuccie \\ Carlo Maria Rotellab \\ ${ }^{a}$ Endocrinology Unit, ${ }^{b}$ Obesity Agency, ${ }^{c}$ Dietetic Unit, ${ }^{d}$ Psychiatric Unit, ${ }^{e}$ Diabetes Agency, \\ AOU Careggi, Florence, Italy
}

\author{
Key Words \\ Obesity $\cdot$ Motivation $\cdot$ Muscle mass $\cdot$ Weight loss
}

\begin{abstract}
Background: In previous pilot studies we have demonstrated that the Treatment Motivation and Readiness Test (TRE-MORE) is capable of predicting the outcome of obesity therapy and that a higher muscle mass (MM) is associated with a greater weight loss. Purposes of the present study were: to confirm the predictive value of TRE-MORE scores and MM, using a standardized non-pharmacologic intervention for weight loss; to explore the relationship between TRE-MORE and MM; to discriminate predictors of attendance from predictors of final therapeutic success. Methods: A consecutive series of 331 patients was enrolled and addressed to a standardized treatment protocol. Results: Mean weight loss at 6 months was $-5.03 \%$. Among participants, $48.7 \%$ lost at least $5 \%$ initial body weight after 6 months and had significantly higher TRE-MORE total scores and MM. Weight loss was significantly associated with baseline MM, TRE-MORE-3, and a lower number of previous diets. Significantly lower TRE-MORE-3 scores were associated with drop-out. Conclusion: The present study confirms that therapeutic success is predicted by TRE-MORE scores and, independently from these, by estimated MM (after adjustment for BMI). TRE-MORE total score is a predictor of failure, but not of attendance, whereas drop-out patients showed a lower score only in TREMORE-3 subscale which investigates lifestyle habits.


Cresci et al.: Fit and Motivated: Outcome Predictors in Patients Starting a Program for Lifestyle Change

\section{Introduction}

The ongoing worldwide obesity epidemic represents a major burden for most healthcare systems [1]. Programs for lifestyle change are time-consuming and require a large amount of resources [2]. At the same time, success rates of therapeutic interventions for weight loss are rather low [3] so that a remarkable effort is spent for the treatment of subjects who will not obtain any relevant benefit. The identification of patients who are most likely to succeed is a possible strategy for focusing existing resources and optimizing therapeutic results [3].

Therefore, the identification of factors possibly associated with successful weight loss could represent an important research area to improve the outcomes of obesity management. A close matching between treatment features and patients' pretreatment needs and/or characteristics could be a pivotal strategy to reduce treatment attrition [4]. In the past, several research trials have investigated the baseline predictors of successful obesity treatment [5-7]. Among pretreatment variables, less previous dieting, fewer weight loss attempts, higher self-motivation, general efficacy, and autonomy have been found to be the best prospective predictors of successful weight management [8]. Finally, baseline weight loss expectations are independent cognitive predictors of attrition in obese patients entering a weight loss program; the higher the expectations, the higher attrition at 12 months [9]. The relative contribution of those factors to therapeutic success as well as their interaction deserve further investigation.

In a previous pilot study, we validated in a consecutive series of patients attending our outpatient clinic the Treatment Motivation and Readiness Test (TRE-MORE), a self-report questionnaire, assessing both the treatment motivation and readiness with good predictive capacity for weight loss [10]. Moreover, we have also found that a higher muscle mass (MM), as estimated through bioimpedance analysis (BIA), is associated with a greater weight loss in obese patients. All patients referred to our outpatient Obesity Agency were enrolled in this study and underwent the standard treatment for obesity proposed in the clinic [11]. These two exploratory studies were performed on small samples of subjects, without a standardized treatment protocol. Furthermore, it is possible that biological parameters (such as MM) and psychological factors (such as those assessed by TRE-MORE) could be related to each other, with highly motivated patients having a healthier lifestyle which determines an increase in $\mathrm{MM}$; this hypothesis should be experimentally verified.

It should also be considered that in randomized trials as well as in epidemiological studies analyses are typically performed by intention to treat, with drop-outs assimilated to failures.

Although it is reasonable that most patients withdrawing from therapeutic programs do not lose weight, it is also possible that some of those patients abandon the scheduled sessions because they have reached their therapeutic target. Furthermore, some of the patients fail to lose weight despite their regular attendance; it is possible that predictors of attendance differ from predictors of final success.

The aims of this interventional study were as follows:

- To confirm the predictive value of TRE-MORE scores and BIA-derived MM for therapeutic success, using a standardized non-pharmacologic intervention for weight loss.

- To explore the relationship between TRE-MORE scores and MM, and their relative independent contribution to the prediction of weight loss.

- To discriminate predictors of attendance from predictors of final therapeutic success. 
Table 1. Inclusion and exclusion criteria of patients
Cresci et al.: Fit and Motivated: Outcome Predictors in Patients Starting a Program for Lifestyle Change
Inclusion criteria

Obesity or overweight (BMI $\geq 27 \mathrm{~kg} / \mathrm{m}^{2}$ )

Age 18-65 years

Residence within $40 \mathrm{~km}$ from the clinic

Informed consent

Exclusion criteria

Patients living at distances more than $40 \mathrm{~km}$

Uncontrolled endocrine disorders, such as hypo- or hyperthyroidism

Diabetes

Pregnancy

Illiteracy, or inadequate knowledge of the Italian language

Any condition interfering with the possibility of regular physical exercise (e.g., severe cardiac dysfunction, severe respiratory insufficiency, major neurologic disorders, etc.)

Diagnosis of major depression, bipolar disorder, obsessivecompulsive disorder, schizophrenia, or mental retardation

Current treatment with antipsychotics, antiepileptics, tricyclic antidepressants, or lithium

Intention to move more than $40 \mathrm{~km}$ far from Florence in the following 12 months
Table 2. Baseline characteristics of patients

\begin{tabular}{ll}
\hline & $\mathrm{n}$ \\
\hline Patients (F/M) & $266(194 / 72)$ \\
\hline & Mean \pm SD \\
\hline Age, years & $43.2 \pm 11.9$ \\
Weight, kg & $105.4 \pm 22.6$ \\
BMI, kg/m² & $38.8 \pm 6.8$ \\
Waist circumference, cm & $118.1 \pm 16.2$ \\
TRE-MORE1 score & $3.1 \pm 0.5$ \\
TRE-MORE2 score & $3.2 \pm 0.4$ \\
TRE-MORE3 score & $2.9 \pm 0.7$ \\
TRE-MORE total score & $3.0 \pm 0.5$ \\
TBW, \% & $44.4 \pm 9.5$ \\
FFM, \% & $60.5 \pm 13.0$ \\
MM, \% & $43.6 \pm 11.3$ \\
FM, \% & $44.6 \pm 13.6$ \\
\hline
\end{tabular}

$\mathrm{F}=$ Female; $\mathrm{M}=$ male

\section{Patients and Methods}

Patients

The study was carried out in the outpatient clinic of the Obesity Agency of the University of Florence. A consecutive series of 331 patients seeking treatment for overweight/obesity between September 1, 2010 and September 30, 2011 was enrolled, provided that they fulfill all of the inclusion criteria and none of the exclusion criteria reported in table 1. Most of the conditions listed in this table were considered exclusion criteria as they could interfere with the efficacy of weight loss programs and/or with compliance to suggested regimens of food intake and physical activity.

The baseline characteristics of the patients are fully described in table 2 . 
Cresci et al.: Fit and Motivated: Outcome Predictors in Patients Starting a Program for Lifestyle Change

Before the collection of data, the procedures of the study were fully explained in the first routine visit; after that, the patients were asked to provide their written informed consent to the participation to the study. The study protocol had been previously approved by the Local Ethical Committee.

\section{Treatment Protocol}

All patients were addressed to a standardized treatment protocol.

At enrollment, the patients were visited by an endocrinologist (B.C., L.P., or M.B.), who provided instructions for physical exercise. At baseline, the patients also met a dietician (R.P. or C.G.) who assessed usual food intake on the basis of a 30-day recall, using an appropriate software (Gedip by Solutions S.n.c, Reggio Emilia, Italy), together with photo atlas for the determination of portions (Istituto Scotti-Bassani, Italy). A goal of a $500 \mathrm{kcal} /$ day reduction from usual food intake was agreed upon with the patient. In order to reach that objective, the patients were asked to self-monitor their food intake for at least a week before each monthly follow-up visit with the dietitian. Reported eating behavior was used as a basis for a personalized educational intervention aimed at modifying eating habits.

\section{Measurements}

At baseline, an endocrinologist collected an accurate medical history, performed a complete physical examination, and measured anthropometric parameters (BMI, waist circumference, blood pressure, and heart rate). Body fat (BF) and lean body mass was estimated through BIA, using a tetrapolar single frequency (50 kHz) phase-sensitive impedance analyzer (QUANTUM/S; AkernSrl, Florence, Italy) as previously described [11]. Measurement were repeated twice, and the average of the two taken as the criterion measure for analysis. MM, total body water (TBW), fat mass (FM), fat-free mass (FFM), and percentage BF were estimated on the basis of impedance and reactance using Bodygram Software 1.31 (AkernSrl). At the same day, the patient underwent a face-to-face short interview with one of two expert psychiatrists (G.C. or V.R.) who formulated psychiatric diagnoses based on DSM-IV criteria [12]. A blood sample was drawn after 12-hour overnight fast for glucose (Beckman Instruments, Fullerton, CA, USA) and thyroid-stimulating hormone (Elettrochemoluminescent, Modular Roche, Milan, Italy) as a screening for diabetes and hypo/hyperthyroidism, which represented exclusion criteria.

Anthropometric measures, including waist circumference and body weight, were again performed after 1, 2, 4, and 6 months; BIA was repeated after 6 months.

At enrolment, while waiting for the first visit with the endocrinologist, patients were asked to complete the TRE-MORE. This short and simple self-reported questionnaire is a predictor of weight loss in patients undergoing specific therapeutic programs [10]; the test provides a global score, together with three subscale scores exploring different domains (TRE-MORE 1: obstacles and desire to overcome; TRE-MORE 2: taking care of themselves and sharing the problems; TRE-MORE 3: current lifestyle). The Italian validated version of the TRE-MORE questionnaire is currently available for other clinicians.

\section{Definition of Outcomes}

Therapeutic success was defined as a weight loss at 6 months of at least $5 \%$ from baseline. In order to assess this outcome, the analysis was performed on an intention-to-treat basis, replacing missing data with the last observation carried forward. A secondary analysis was performed by protocol, i.e. in those patients who completed the therapeutic program.

A secondary outcome considered was completion of the therapeutic program, defined as attendance to all four follow-up sessions described above. Those patients who did not fulfill these criteria were considered as 'drop-outs'.

\section{Statistical Analysis}

Continuous variables were reported as mean \pm standard deviations (SD), whereas categorical variables were reported as absolute number and percentage. Between-group comparisons of continuous variables were performed using unpaired Student's t test and Mann-Whitney U test for variables with normal and nonnormal distribution, respectively. Correlation analyses were performed with Spearman's method. A stepwise linear multiple regression was applied for the analysis of predictors of BMI reduction across time. Logistic regression analyses were applied for dichotomous outcomes (success vs. failures).

All analyses were performed using SPSS for Windows 15.0 (Chicago, IL, USA). 
Cresci et al.: Fit and Motivated: Outcome Predictors in Patients Starting a Program for Lifestyle Change

Fig. 1. Study flow-chart.

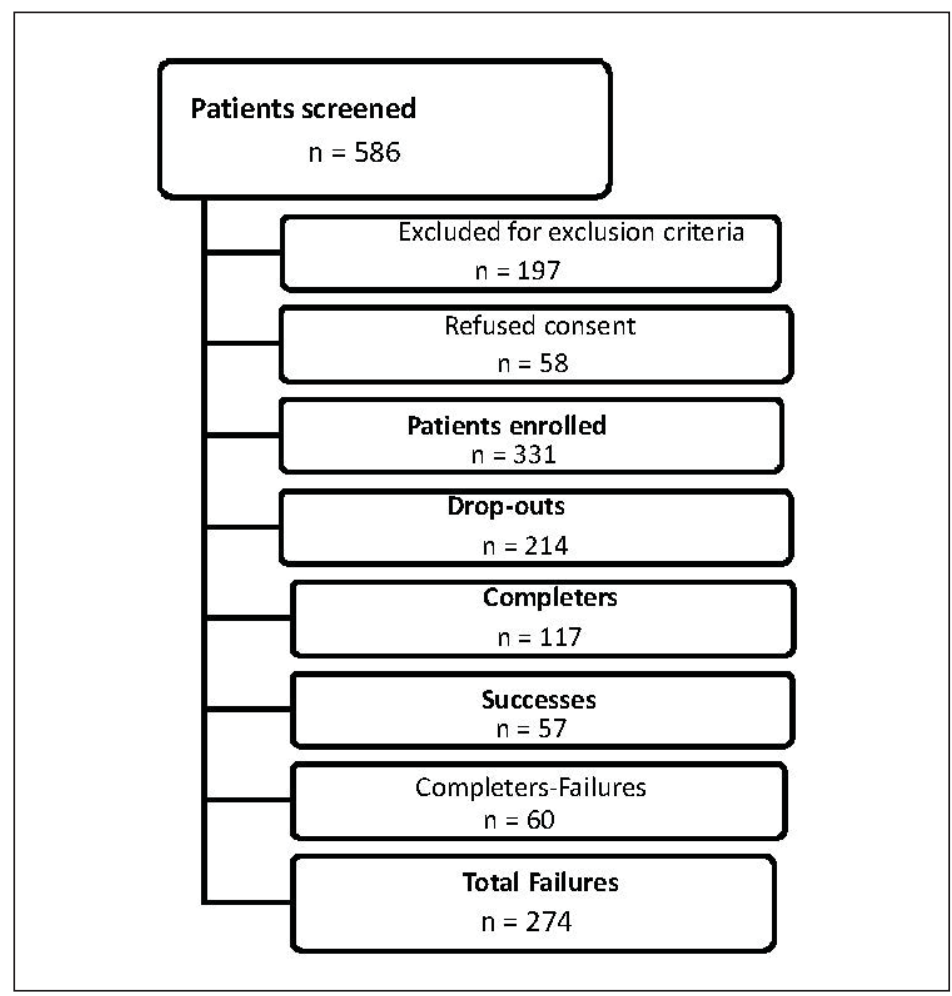

\section{Results}

Treatment Outcomes

Of the enrolled 331 patients, only 117 (35.3\%) completed the 6-month protocol. On the basis of intention-to-treat analysis, mean weight loss at 6 months was $-5.03 \%( \pm 5.2 \%)$. Among participants, $48.7 \%(n=57)$ lost at least $5 \%$ initial body weight after 6 months (fig. 1$)$. A significant $(\mathrm{p}<0.0001)$ decrease was also observed for waist circumference $(127 \pm 15.7 \mathrm{~cm}$ vs. $125 \pm 15.2 \mathrm{~cm}$ and $114.5 \pm 14.8 \mathrm{~cm}$ vs. $111.3 \pm 12.9 \mathrm{~cm}$ in men and women, respectively), FM (35.5 $\pm 7.8 \%$ vs. $30.7 \pm 9.7 \%$ and $43.8 \pm 5.6 \%$ vs. $38.8 \pm 12.4 \%$ in men and women, respectively), and FFM ( $63.6 \pm 7.9 \%$ vs. $66.7 \pm 7.1 \%$ and $56.3 \pm 5.7 \%$ vs $58.2 \pm 6.6 \%$ in men and women, respectively). Moreover, a significant increase $(\mathrm{p}=0.008)$ was observed for MM $(39.5 \pm 6.1 \%$ vs. $42.0 \pm 8.2 \%)$ in women only.

\section{Predictors of Outcome: Weight Loss at 6 Months}

Differences between successful (completers losing at least $5 \%$ of initial body weight at the intention-to-treat analysis) and unsuccessful participants (completers losing less than $5 \%$ of initial body weight and drop-outs) are summarized in table 3 . Waist circumference was higher in successful participants than in those who did fail when considering the whole group of patients ( $122.29 \pm 16 \mathrm{~cm}$ vs. $115.34 \pm 14.5 \mathrm{~cm}$; $\mathrm{p}=0.02)$, while no significant differences could be observed when comparing men and women separately (men successes vs men failures: $133.7 \pm 16 \mathrm{~cm}$ vs. $125 \pm 12 \mathrm{~cm}$ in men, $114 \pm 11.5 \mathrm{~cm}$ vs. $113.7 \pm 14 \mathrm{~cm}$ in women). Furthermore, patients with therapeutic success had significantly higher TRE-MORE total scores as well as higher TBW, FFM, and MM. None of these differences was retained at multivariate analysis (logistic regression), after adjusting for age, sex, and BMI. When 6-month weight loss was considered as a continuous variable, a significant correlation was observed 
Cresci et al.: Fit and Motivated: Outcome Predictors in Patients Starting a Program for Lifestyle Change

Table 3. Differences between successes (losing at least $5 \%$ of initial body weight at the intention-to-treat analysis) and failures (completers losing less than $5 \%$ of initial body weight and drop-outs) ${ }^{\mathrm{a}}$

\begin{tabular}{llll}
\hline Parameters & Successes & Failures & p value $^{\text {b }}$ \\
\hline Sex M/F & $24 / 33$ & $9 / 50$ & 0.874 \\
Age, years & $45.7 \pm 11.2$ & $44.6 \pm 11.4$ & 0.59 \\
BMI, kg/m & $40 \pm 7.3$ & $37.8 \pm 6.3$ & 0.08 \\
Number of previous diets & $1.5 \pm 0.9$ & $1.8 \pm 0.9$ & 0.179 \\
Waist circumference, cm & $122.3 \pm 16$ & $115.3 \pm 14.5$ & 0.020 \\
TRE-MORE1 score & $3.1 \pm 0.5$ & $2.9 \pm 0.5$ & 0.142 \\
TRE-MORE2 score & $3.3 \pm 0.50$ & $3.1 \pm 0.4$ & 0.176 \\
TRE-MORE3 score & $3.2 \pm 0.8$ & $2.9 \pm 0.6$ & 0.140 \\
TRE-MORE total score & $3.1 \pm 0.3$ & $2.9 \pm 0.3$ & 0.008 \\
TBW, \% & $47.3 \pm 11.3$ & $42.9 \pm 7.9$ & 0.020 \\
FFM, \% & $64.2 \pm 15.7$ & $58.3 \pm 10.6$ & 0.020 \\
MM, \% & $46.3 \pm 13.2$ & $41.1 \pm 10.3$ & 0.023 \\
FM, \% & $46.5 \pm 14.4$ & $42.8 \pm 13.2$ & 0.154 \\
\hline
\end{tabular}

$\mathrm{F}=$ Female; $\mathrm{M}=$ male.

${ }^{\mathrm{a}}$ Data are expressed as mean $\pm \mathrm{SD}$. ${ }^{\mathrm{b}}$ Significant differences are given in italics.

Table 4. Differences between drop-outs and completers ${ }^{\mathrm{a}}$

\begin{tabular}{llll}
\hline Parameters & Drop-outs & Completers & p value $^{\mathrm{b}}$ \\
\hline Sex M/F & $38 / 111$ & $34 / 83$ & 0.517 \\
Number of previous diets & $1.8 \pm 1.0$ & $1.7 \pm 0.9$ & 0.33 \\
Waist circumference, cm & $117.6 \pm 16.5(89-154)$ & $118.7 \pm 15.84$ & 0.59 \\
BMI, kg/m & $38.8 \pm 6.8(27-65)$ & $38.8 \pm 6.9(27-62)$ & 0.96 \\
Age, years & $41.7 \pm 12.2(18-65)$ & $45.1 \pm 11.3(19-64)$ & 0.01 \\
TRE-MORE1 score & $3.1 \pm 0.5(1.5-4.2)$ & $3 \pm 0.5(2.0-4.2)$ & 0.34 \\
TRE-MORE2 score & $3.2 \pm 0.4(1.9-4.6)$ & $3.2 \pm 0.5(2.0-4.4)$ & 0.71 \\
TRE-MORE3 score & $2.8 \pm 0.6(1.0-4.7)$ & $3.1 \pm 0.7(1.2-5.0)$ & 0.01 \\
TRE-MORE total score & $3 \pm 0.3(2.1-4.0)$ & $3.0 \pm 0.3(2.1-3.8)$ & 0.82 \\
TBW, \% & $43.7 \pm 9.1(29.5-75.1)$ & $45.1 \pm 9.9(32.9-79.9)$ & 0.24 \\
FFM, \% & $59.8 \pm 12.5(40.3-102.7)$ & $61.3 \pm 13.6(45.0-109.2)$ & 0.35 \\
MM, \% & $43.5 \pm 10.8(26.4-88.5)$ & $43.7 \pm 12(28.9-97.7)$ & 0.88 \\
FM, \% & $44.7 \pm 13.4(22.6-86.2)$ & $44.4 \pm 13.9(17.3-79.4)$ & 0.88
\end{tabular}

$\mathrm{F}=$ Female; $\mathrm{M}=$ male .

${ }^{a}$ Data are presented as mean \pm SD (range). ${ }^{b}$ Significant differences are given in italics.

with FFM ( $\mathrm{r}=0.19, \mathrm{p}=0.03)$ and MM ( $\mathrm{r}=0.22, \mathrm{p}=0.01)$. At multivariate analysis (linear regression), after adjusting for gender, age, waist and BMI, weight loss was significantly associated with higher MM $\left(\mathrm{R}^{2}=0.081 ; \beta=0.268 ; \mathrm{p}=0.037\right)$ and higher TRE-MORE 3 scores $\left(R^{2}=0.067 ; \beta=0.200 ; p=0.050\right)$ at baseline and a lower number of previous diets $\left(R^{2}=0.115\right.$; $\beta=-0,219 ; p=0.031)$, but not with baseline TBW $\left(R^{2}=0.07 ; \beta=0.24 ; p=0.08\right)$.

\section{Predictors of Outcome: Drop-Outs}

Differences between drop-outs and completers are summarized in table 4. Lower TRE-MORE 3 scores were associated with drop-out; this association was confirmed at multi- 
Cresci et al.: Fit and Motivated: Outcome Predictors in Patients Starting a Program for Lifestyle Change

variate analysis (logistic regression: $\mathrm{B}=-0,540 ; \mathrm{p}=0.030$ ) after adjusting for age, sex and BMI. No other significant difference was observed between drop-outs and completers.

When considering the completers group only, TRE-MORE 3 scores $(p=0.05)$ and baseline MM ( $p=0.03)$ were significantly associated with weight loss at 6 months after adjusting for age, sex, and BMI.

\section{Discussion}

The present study confirms our previous findings in smaller samples, reporting that therapeutic success is predicted by TRE-MORE scores and by estimated MM. In comparison with our previous works $[10,11]$, in this study treatment outcome was assessed using a standardized intervention protocol in order to improve the reproducibility of results. Furthermore, the two parameters (TRE-MORE and MM) appear to be independent predictors of therapeutic success.

The TRE-MORE was specifically designed to identify obese patients who were more likely to succeed in weight loss programs [10]. This test includes items related to different areas and, most notably, motivational readiness, self-efficacy, and current lifestyle habits. Although other more complex instruments and more accurate measures are available for each of these domains, the questionnaire has the advantage of providing a simple and easy-to-use tool for predicting therapeutic success.

We show here that, regardless of the score of TRE-MORE, estimated MM is also an independent predictor of weight loss. As expected, patients with higher skeletal MM had a better success rate, probably due to higher energy expenditure. Patients with higher baseline BMI, who can reach more easily the pre-defined target of $5 \%$ weight loss, are expected to have a lower percent MM. Despite this fact, the proportion of MM at enrolment was a positive, and not a negative, predictor of success. On the other hand, MM is lower in women, who seem to have a lower success rate; therefore, the possibility that the association of higher MM with weight loss is at least partly due to the effect of gender should be considered. However, in multivariate analysis, adjustment for gender attenuated, without completely eliminating, the association of baseline MM with weight loss. Furthermore, MM (in women) was shown to be significantly increased after the 6-month protocol. This finding could be related to the fact that a specific feature of the program applied in the present study was the focus on physical activity, which received a particular emphasis throughout the protocol. In fact, the majority of the patients enrolled started exercising regularly during treatment and maintained this habit during follow-up. Starting regular physical exercise in previously sedentary individuals can even determine a temporary increase in body weight in some individuals due to increased skeletal MM[15]. On the other hand, it has to be pointed out that these patients effectively lose mainly fat mass, as indirectly demonstrated also by the significantly reduced waist circumference at 6 months.

However, the study presented here has some limitations. First of all, the measurement of body composition poses remarkable methodological problems, with the most accurate tools (such as magnetic resonance imaging or DEXA) being inapplicable in large-scale studies or in routine clinical practice. BIA is a simpler alternative; although less reliable than other measurements [13], this instrument provides estimates which are sufficient for predicting treatment outcomes $[11,14]$. Furthermore, the definition of success was based on an intentionto-treat analysis, considering drop-outs as failures. This is a conservative approach, underestimating the effects of therapy; in fact, it is possible that some of the patients who withdrew during the treatment program had already reached their desired goals. 
Cresci et al.: Fit and Motivated: Outcome Predictors in Patients Starting a Program for Lifestyle Change

\section{Conclusions}

Failure in treatment programs can be due to early withdrawal or to insufficient weight loss despite adherence to planned visits. The results of the present study seem to confirm the hypothesis that these two outcomes which are usually combined in the definition of treatment failure have a different genesis and thus different predictors. Therefore, MM, which is a predictor of overall failure, is not related to drop-out, which means that patients with a lower $\mathrm{MM}$ are more likely to have an unsatisfactory weight loss despite attendance to the entire planned program. Furthermore, the TRE-MORE total score is a predictor of failure, as previously reported [10], but not of drop-out, whereas patients withdrawing prematurely showed a lower score in the TRE-MORE 3 subscale which investigates lifestyle habits. It can be speculated that patients with an unhealthier lifestyle at baseline should make greater modifications to their habits and, therefore, could be at higher risk of abandoning the program.

The use of different instruments to identify patients with a greater chance of success can be useful for allocating available resources to those who are more likely to benefit. Moreover, the identification of predictors is also useful for a better understanding of barriers to change, which could be overcome by more specifically targeting therapeutic interventions. For example, it is conceivable that lack of motivation could be countered by motivational interviews and reduced MM by appropriate exercise programs. The reliability of the parameters delineated here to predict weight loss program outcomes in some subpopulations of patients needs to be verified by specifically designed randomized clinical trials.

\section{Acknowledgements}

The present study was supported by grants to CMR by the Italian Ministry of Health (Rome, Italy), by the Regione Toscana Department of Health (Florence, Italy), by Ely Lilly Italy and by Fondazione Cassa di Risparmio di Firenze, Italy.

\section{Disclosure Statement}

For this study the authors declare no conflict of interest. For this study CMR received unconditioned grants from Ely Lilly Italy and from Fondazione Cassa di Risparmio di Firenze, Italy.

\section{References}

$>1$ Hedley AA, Ogden CL, Johnson CL, Carroll MD, Curtin LR, Flegal K: Prevalence and trends in obesity among US adults, 1999-2000. JAMA 2004;291:2847-2850.

2 World Health Organization: Obesity: Preventing and Managing the Global Epidemic. Geneva, World Health Organization, 1998.

3 Teixeira PJ, Going SB, Houtkooper LB, Cussler EC, Metcalfe LL, Blew RM, Sardinha LB, Lohman TG: Pretreatment predictors of attrition and successful weight management in women. Int J Obes Relat Metab Disord 2004;28:1124-1133.

4 Cresci B, Rotella CM: Motivational readiness to change in lifestyle modification programs. Eat Weight Disord 2009;14:158-162.

5 Brownell KD: Behavioral, psychological, and environmental predictors of obesity and success at weight reduction. Int J Obes 1984;8:543-550.

-6 Foreyt JP, Goodrick GK: Factors common to successful therapy for the obese patient. Med Sci Sports Exerc 1991;23:292-297.

7 Wadden TA, Letizia KA: Predictors of attrition in weight loss in patients treated by moderate and severe caloric restriction; in Wadden TA, VanItallie TB (eds): Treatment of the Seriously Obese Patients. New York, Guilford Press, 1992, pp 383-410 
8 Teixeira PJ, Going SB, Sardinha LB, Lohman TG: A review of psychosocial pre-treatment predictors of weight control. Obes Rev 2005;6:43-65.

-9 Dalle Grave R, Calugi S, Molinari E, Petroni ML, Bondi M, Compare A, Marchesini G; QUOVADIS Study Group: Weight loss expectations in obese patients and treatment attrition: an observational multicenter study. Obes Res 2005;13:1961-1969.

10 Cresci B, Castellini G, Pala L, Ravaldi C, Faravelli C, Rotella CM, Ricca V: Motivational readiness for treatment in weight control programs: the TREatmentMOtivation and REadiness (TRE-MORE) test. J Endocrinol Invest 2011;34:e70-77.

11 Dicembrini I, Pala L, Cresci B, Cremasco F, Rotella CM: Predictors of weight loss in the clinical management of obese patients: the relevance of body composition. Obes Metab 2010;1:29-33.

12 American Psychiatric Association: Diagnostic and Statistical Manual of Mental Disorders. Fourth Edition (DSM-IV). Washington DC, American Psychiatric Association, 1994.

13 Kyle UG, Bosaeus I, De Lorenzo AD: Bioelectrical impedance analysis: part I - review of principles and methods. Clin Nutr 2004;23:1226-1243.

14 Deurenberg P, Andreoli A, Borg P, Kukkonen-Harjula K, de Lorenzo A, van Marken Lichtenbelt WD, Testolin G, Vigano R, Vollaard N: The validity of predicted body fat percentage from BMI and from impedance in samples of five European populations. Eur J Clin Nutr 2001;55:973-979.

15 Schmitz KH, Jensen MD, Kugler KC, Jeffery RW, Leon AS: Strength training for obesity prevention in midlife women. Int J Obes Relat Metab Disord 2003;27:326-333. 